\title{
Heart rate variability in bipolar disorder and borderline personality disorder: a clinical review
}

\author{
Oliver Carr, ${ }^{1}$ Maarten de Vos, ${ }^{1}$ Kate E A Saunders ${ }^{2,3}$
}

${ }^{1}$ Institute of Biomedical Engineering, University of Oxford, Oxford, UK; ${ }^{2}$ University of Oxford Department of Psychiatry, Warneford Hospital, Oxford, UK; ${ }^{3}$ Oxford Health NHS Foundation Trust, Warneford Hospital, Oxford, UK

Correspondence to Dr Kate E A Saunders, University of Oxford Department of Psychiatry, Warneford Hospital, Oxford, UK; kate. saunders@psych.ox.ac.uk

\section{ABSTRACT}

Heart rate variability (HRV) in psychiatric disorders has become an increasing area of interest in recent years following technological advances that enable non-invasive monitoring of autonomic nervous system regulation. However, the clinical interpretation of HRV features remain widely debated or unknown. Standardisation within studies of HRV in psychiatric disorders is poor, making it difficult to reproduce or build on previous work. Recently, a Guidelines for Reporting Articles on Psychiatry and Heart rate variability checklist has been proposed to address this issue. Here we assess studies of HRV in bipolar disorder and borderline personality disorder against this checklist and discuss the implication for ongoing research in this area.

\section{INTRODUCTION}

Heart rate variability (HRV) is the variation in time interval between each heart beat, recorded as the R-R interval of an ECG signal. It is a complex physiological phenomenon that results from the modification of the heart rate (HR) by respiratory, circulatory, autonomic, endocrine and mechanical factors. Reductions in HRV are associated with a range of conditions such as diabetic neuropathy, sepsis and following myocardial infarct but have become of increasing interest in psychiatry because of the link between autonomic dysfunction and psychiatric illness. Changes in HRV have been reported in a range of mental disorders ${ }^{1}$ as well as correlating with psychological dimensions such as social cognition, ${ }^{2}$ executive function ${ }^{3}$ and emotional regulation. ${ }^{4}$ The higher incidence of cardiovascular disease associated with some psychiatric disorders has also led to greater focus on autonomic system function. Both bipolar disorder (BD) and borderline personality disorder (BPD) have higher rates of cardiovascular mortality. Despite their contrasting aetiologies and treatments, they are phenotypically similar, both being characterised by mood instability. This makes them ideal groups in which to compare HRV (figure 1).

Variations in HR occur due to the constant need of the heart to adapt to changing circumstances, and it is thought that loss of balance of the sympathetic and parasympathetic nervous systems causes an alteration to the structure of HRV. ${ }^{56} \mathrm{HRV}$, as a measure of nervous system balance, can therefore provide a quantification of physiological changes associated with mental health disorders, with many studies investigating these associations through a number of time domain, frequency domain and non-linear methods of quantifying HRV. ${ }^{7-9}$

Initial studies of HRV used relatively simple linear algorithms to quantify variability and autonomic function, either in the time domain or the frequency domain. ${ }^{7}$ Later studies suggested that interactions between the autonomic nervous system and the regulation of the cardiovascular system may be non-linear, with more complex non-linear algorithms potentially providing better metrics to quantify these interactions. In addition, non-linear measures are often less affected by non-stationarity of signals, whereas linear methods require stationary signals. ${ }^{10}$

The linear time domain features are the most straightforward metrics of HRV to calculate. These measures are statistical calculations of the intervals between successive normal complexes (see table 1). Frequency domain features are derived using spectral analysis. This approach provides information about how the variance (or power) is distributed as a function of frequency. These tend to be applied either to short stationary recordings ( $5 \mathrm{~min}$ ) or over 24-hour periods, with frequency metrics being linked to levels of sympathetic and primarily parasympathetic activity in the autonomic nervous system (ANS). ${ }^{11}$
Non-linear features that have become more widely used include: measures of entropy (sample entropy and approximate entropy), which measure irregularity and randomness of signals; detrended fluctuation analysis, to distinguish between short-term internal variations and longer term variations; power law exponent, which determines the fractal nature of the interbeat interval signal; and recurrence quantification analysis, a method to quantify repeating instances of signals, as well as algorithms such as Teager-Kaiser energy and Lempel-Ziv complexity. Although these non-linear methods of quantifying HRV may give more insight into the complex interactions linked to the cardiovascular system, it is much more difficult to give clinical interpretation to their metrics. ${ }^{812} 13$

Studies involving HRV in psychiatry illness have minimal standardisation, especially in the timescales and methods of recording ECG data. The majority of studies obtain ECG recordings from a 5 min period and calculate HRV measures on the 5 min R-R interval signal. However, as much longer recordings are becoming easier to obtain, HRV measures can be calculated over much longer periods, making comparison between HRV measures on different timescales difficult. In addition to the varying timescales of recordings, the activity levels of the participants and method of data collection may also differ. For example, data are often collected when the participant is 'at rest'; however, the posture of the participant, time of day, recently eating or drinking and many other factors may all contribute to variations in HRV. Stimuli, in the form of images or exercises, may also be given during the recordings, again making comparison of HRV measures between studies difficult. Development of devices allowing ambulatory monitoring of ECG over periods of hours or even days enable participants to continue with their regular activity and behavioural patterns while being recorded. While these data can be recorded in a less clinical and more natural manner, it becomes increasingly difficult to monitor all external factors that considerably alter the behaviour of the cardiovascular system. For studies involving mental health or investigating difference in HRV between groups, it is often not possible to determine whether differences are due to the different diagnoses or due to the external influences.

There are no widely agreed standard measures for HRV quantification and the clinical interpretation of many HRV features remain widely debated or unknown. Quintana et al ${ }^{14}$ provided recommendations to improve HRV research in psychiatry and introduced a Guidelines for Reporting Articles on Psychiatry and Heart rate variability (GRAPH) checklist for good practice to make an attempt to standardise reporting in these studies. The guidelines consist of four main areas: selection of participants, interbeat interval collection, data analysis and cleaning and HRV calculation. Within each topic area a list of checklist items is defined, as shown in table 2. 


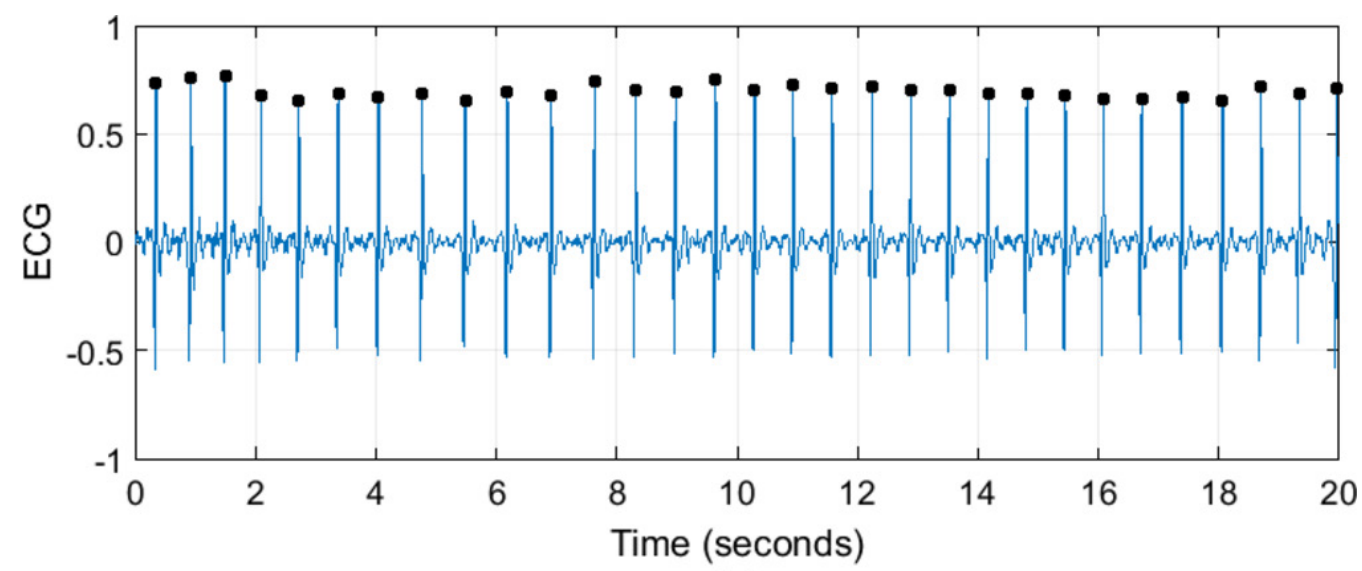

(a)

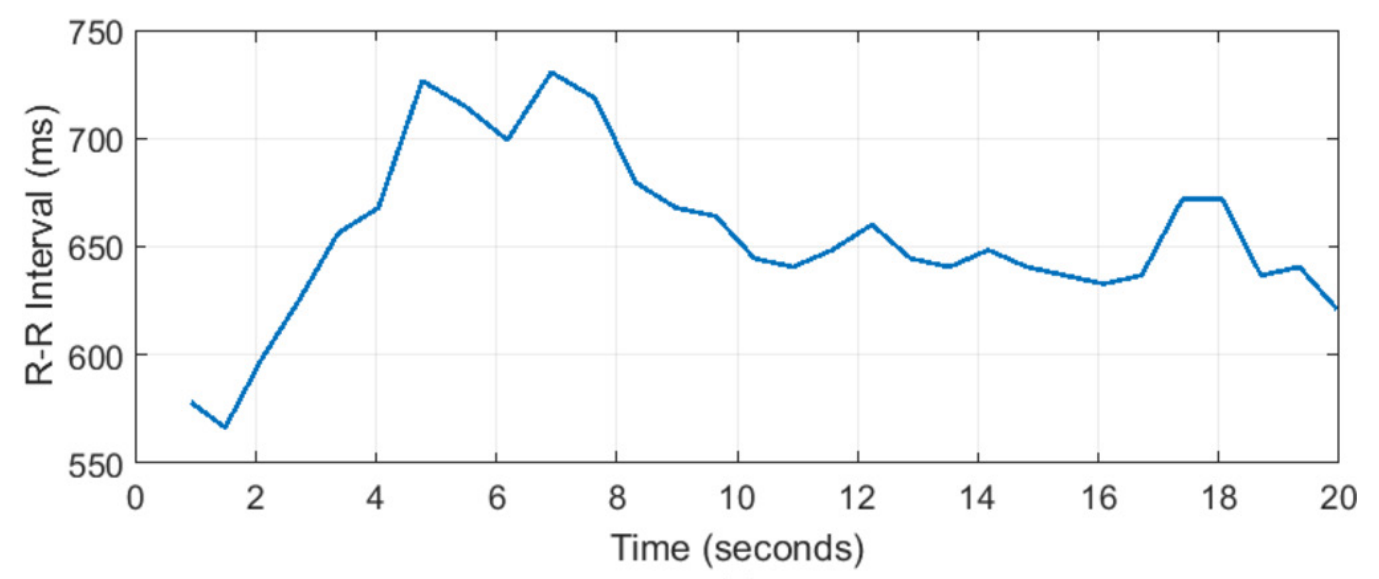

(b)

Figure 1 (A) An ECG signal with R peaks denoted by black dots. (B) The corresponding tachogram or $R-R$ interval series.

Here we review HRV in BD and BPD making specific reference to the good practice checklist. BPD and BD are two psychiatric diagnoses that despite differing aetiologies have a shared phenotype; both disorders are associated with mood instability, impulsivity, suicidal behaviour and low mood. Comparing HRV in the two disorders allows us to explore whether the shared phenotype is associated with similar or differing underlying physiology.

\section{METHODS}

To identify relevant evidence of HRV in mood disorders, we searched PubMed, PsycINFO, Google Scholar and the Cochrane Library for papers published between 1980 and May 2017. No language constraints were applied, and the following key words were used: depression, bipolar disorder, borderline personality disorder and heart rate variability. This search was supplemented by hand search of references from articles in the initial search. Articles were included in which ECG recordings were analysed from at least one participant diagnosed with either BD or BPD.

The methods, results and interpretation of each article were summarised, and their adherence to the GRAPH checklist was examined.

\section{PRESENTATION \\ HRV in BD}

A range of studies of HRV in patients suffering from BD have been conducted but are often difficult to compare due to the BD participants being in varying mood states across the studies. Statistical comparisons are often made between the psychiatric disorder groups and a control group and occasionally between participants in different mood states. The earliest study was carried out by Cohen et al. ${ }^{7}$ ECG from 39 euthymic bipolar patients and 39 healthy controls were recorded in a controlled environment. Time domain and spectral analysis were performed on the $\mathrm{R}-\mathrm{R}$ intervals, with bipolar patients having lower root mean square of successive differences (RMSSD), lower total power, higher nHF and an increased low frequency to high frequency (LF/HF) ratio, independent of any medication taken. The features calculated were defined slightly differently to the standard methods and with no information given about the length of time of ECG recording thus making comparisons with this study difficult. Further work was carried out on the same participants in 2005 by Todder et al using non-linear analysis; however, they found no significant differences between the euthymic $\mathrm{BD}$ and healthy groups using the non-linear features that include: Lyapunov exponent, Shannon entropy and Poincaré plots. ${ }^{15}$

Other studies carried out on euthymic bipolar patients compared with healthy controls include the study by Gruber et al in 2011. Here, the emotional responses of $23 \mathrm{BD}$ participants were compared with that of 24 healthy controls after various stimuli; the study found greater HRV in the BD group after the stimuli through an increase in measures related to parasympathetic activity. ${ }^{16}$ More recently, in 2015, Voggt et al investigated HRV features in 90 euthymic bipolar patients compared with 62 healthy controls. Parameters were calculated from a 30 min ECG recording, with SD of R-R interval (SDNN), low frequency (LF) power and high frequency (HF) power found to be lower in the BD group. ${ }^{17}$ In 2012 , Levy used several physiological measures of autonomic nervous system function to determine differences between patients with $B D$ and healthy controls, without using the traditional HRV features. Thirty-three patients with BD and 22 healthy controls had 5 min of ECG recorded at rest, with significant differences found in physiological HR features between patients with $\mathrm{BD}$ and healthy controls. ${ }^{18}$ 
Table 1 The most widely used HRV measures used in the literature with short interpretation of their meanings

\begin{tabular}{|c|c|c|c|}
\hline HRV measure & Units & Domain & Description \\
\hline Mean of R-R intervals (mRR) & ms & Time domain & A measure of average $R-R$ interval (60/heart rate). \\
\hline Standard deviation (SD) of R-R intervals (SDNN) & $\mathrm{ms}$ & Time domain & A measure of variability of $R-R$ intervals across the whole signal. \\
\hline SD of successive differences & ms & Time domain & $\begin{array}{l}\text { A longer term measure of variability through } S D \text { of differences between adjacent } R-R \\
\text { intervals. }\end{array}$ \\
\hline SD of average $R-R$ intervals & ms & Time domain & $\begin{array}{l}\text { For longer signals, } \mathrm{mRR} \text { is calculated on segments, often } 5 \mathrm{~min} \text {, and SD of these values are } \\
\text { calculated. }\end{array}$ \\
\hline Percentage of $\mathrm{R}-\mathrm{R}$ intervals over $\mathrm{x}$ ms & $\%$ & Time domain & As above, normalised to the total number of intervals. Can be used when signal lengths vary. \\
\hline Total power (TP) & $\mathrm{ms}^{2}$ & Frequency domain & $\begin{array}{l}\text { Total power in the frequency spectra up to } 0.4 \mathrm{~Hz} \text {. Can be measured from zero, from the start } \\
\text { of the VLF band }(0.003 \mathrm{~Hz}) \text { or from the } L F \text { band }(0.04 \mathrm{~Hz}) \text {. }\end{array}$ \\
\hline Very low frequency power (VLF) & $\mathrm{ms}^{2}$ & Frequency domain & Power in the $0.003-0.04 \mathrm{~Hz}$ frequency band. \\
\hline Low frequency power (LF) & $\mathrm{ms}^{2}$ & Frequency domain & $\begin{array}{l}\text { Power in the } 0.04-0.15 \mathrm{~Hz} \text { frequency band. Often linked to combined levels of sympathetic } \\
\text { and parasympathetic activity; however, this interpretation is widely debated. }\end{array}$ \\
\hline Low frequency to high frequency (LF/HF) ratio & - & Frequency domain & $\begin{array}{l}\text { Ratio between LF and HF power bands. Often associated with sympathovagal balance in the } \\
\text { literature; however, this interpretation is also debated. }\end{array}$ \\
\hline Sample entropy & - & Non-linear & $\begin{array}{l}\text { Entropy measures periodic variations in the R-R interval signals not detectable using means } \\
\text { and SD. }\end{array}$ \\
\hline Detrended fluctuation analysis exponent () & - & Non-linear & $\begin{array}{l}\text { Finds long-term correlations in the signal, with the exponent giving a value of self-correlation } \\
\text { of the signal. }\end{array}$ \\
\hline Poincaré standard deviations (SD1, SD2) & - & Non-linear & $\begin{array}{l}\text { Poincaré plots plot } R-R \text { intervals against the succeeding } R-R \text { intervals. With the } S D \text { in } \\
y=x \text { representing longer term variation and in the perpendicular direction, short-term } \\
\text { variation. }\end{array}$ \\
\hline pNN50 & - & Time domain & $\begin{array}{l}\text { Proportion of consecutive R-R intervals that differ by more than } 50 \mathrm{~ms} \text {. } \\
\text { Measure of parasympathetic activity. }\end{array}$ \\
\hline
\end{tabular}

HRV, heart rate variability.

Lee et $\mathrm{l}^{19}$ investigated the differences in HRV between $33 \mathrm{BD}$ patients with subsyndromal depressive symptoms and 59 healthy controls. A 5 min section of ECG was recorded on each participant in a controlled rest situation, with HRV features calculated in the time and frequency domains. Significantly lower values of SDNN, pNN50, total power and very low frequency (VLF) power were found in patients with BD. Negative correlations were also found between the scores from the depression questionnaires and a large range of time and frequency domain features using Pearson correlation: SDNN $(r=-0.415, p=0.016)$, RMSSD $(r=-0.347, p=0.048), p N N 50(r=-0.436, p=0.011), L F(r=-0.379$, $p=0.03)$ and $H F(r=-0.396, p=0.022)$.

The focus of other studies has been on differences in HRV in bipolar depression compared with healthy controls; work by Chang et al20 in 2015 compared HRV between these two groups and also between patients with unipolar depression (UD). One hundred and sixteen patients with bipolar depression, 591 physically healthy patients with UD and 421 healthy controls were included in the study, with interviews and self-reported questionnaire scores used as a measure of depression, and 5 min ECG was recorded in lying position after 20 min of rest. Compared with UD, bipolar depression was associated with significantly lower time domain features, along with significantly lower LF and HF power; however, the LF-to-HF ratio was significantly higher in bipolar depression. Comparing the two depressed groups to the healthy controls, features indicative of parasympathetic activity were significantly lower in both groups compared with healthy controls, whereas features supposedly related to sympathetic activity were significantly higher in bipolar depression than in healthy controls, but $U$ participants and healthy controls showed no difference in these features. Basset et a ${ }^{21}$ studied 29 BD participants who had been well for at least the last 3 months and compared linear time and frequency domain measures of HRV to 41 participants with major depressive disorder and 38 healthy controls during sleep. The RMSSD of $\mathrm{R}-\mathrm{R}$ intervals was found to be significantly lower in BD compared with healthy participants, with major depressive disorder participants having significantly reduced HRV compared with healthy controls through the majority of metrics. They suggest ANS dysfunction in BD during sleep through a reduction in parasympathetic activity.

Investigations into differences between HRV in patients with $\mathrm{BD}$ in a manic state and healthy controls have also been performed. Henry et a ${ }^{12}$ carried out a study on 23 manic BD patients, 14 patients with schizophrenia and 23 healthy controls. HRV was quantified through time domain, frequency domain and non-linear analysis on 5 min of ECG when the participant was at rest. Patients with BD showed lower RMSSD, pNN50 and nHF values and an increase in LF/HF ratio, with indications of reduced parasympathetic activity in patients with $\mathrm{BD}$ compared with healthy controls through lower HF power. Reductions in the entropy of the ECG signal were also significant in patients with BD, suggesting reduced HRV. Chang et $a^{22}$ also investigated HRV in manic BD patients compared with healthy controls. Sixty-one unmedicated patients with $\mathrm{BD}$ and 183 healthy controls had a 5 min section of ECG recorded at rest, and significantly reduced $\mathrm{mRR}$, SDNN, LF power and $\mathrm{HF}$ power was found in the manic BD group, suggesting ANS dysregulation in mania.

A study investigating differences in HRV in $\mathrm{BD}$, schizophrenia and healthy controls was performed in 2015 by Quintana et $\mathrm{al}^{9}$, which included 33 patients with $\mathrm{BD}, 47$ patients with schizophrenia and 212 healthy controls; however, no information is given about the current mental state of the BD group patients. HRV was found to be reduced in both the disorders compared with healthy controls through decreased mean of $R-R$ intervals (mRR) and 
Table 2 Guidelines for Reporting Articles on Psychiatry and Heart rate variability $^{14}$

\begin{tabular}{|c|c|c|}
\hline Topic & Number & Checklist Item \\
\hline Participant selection & 1 & \\
\hline Psychiatric group selection & 1a & $\begin{array}{l}\text { Recruitment details and assessment } \\
\text { methods }\end{array}$ \\
\hline Control group selection & $1 b$ & $\begin{array}{l}\text { Recruitment details and methods to rule out } \\
\text { psychiatric illness }\end{array}$ \\
\hline Inclusion criteria & 1c & $\begin{array}{l}\text { Description of criteria (eg, absence of } \\
\text { physical health problems) }\end{array}$ \\
\hline Disease characteristics & $1 d$ & $\begin{array}{l}\text { Description of disease duration, severity, } \\
\text { comorbidities and medication }\end{array}$ \\
\hline Demographics & $1 \mathrm{e}$ & $\begin{array}{l}\text { Details on age, gender, physical activity } \\
\text { levels, smoking and so on }\end{array}$ \\
\hline $\mathrm{R}-\mathrm{R}$ interval collection & 2 & \\
\hline Hardware/software details & $2 a$ & Brand and electrode configuration \\
\hline $\begin{array}{l}\mathrm{R}-\mathrm{R} \text { interval collection } \\
\text { details }\end{array}$ & $2 b$ & $\begin{array}{l}\text { Sampling rate, length of data, time of day, } \\
\text { filtering, posture and participant instructions }\end{array}$ \\
\hline Data analysis and cleaning & 3 & \\
\hline $\mathrm{R}-\mathrm{R}$ interval calculation & 3a & $\begin{array}{l}\text { R-R interval calculation and resampling } \\
\text { methods }\end{array}$ \\
\hline $\begin{array}{l}\mathrm{R}-\mathrm{R} \text { interval artefact } \\
\text { identification }\end{array}$ & $3 b$ & $\begin{array}{l}\text { R-R interval artefact identification method } \\
\text { (eg, algorithm and manual inspection) }\end{array}$ \\
\hline $\mathrm{R}-\mathrm{R}$ interval data loss & $3 c$ & $\begin{array}{l}\text { Reasons for loss (eg, ectopy and equipment } \\
\text { failure) }\end{array}$ \\
\hline $\mathrm{R}-\mathrm{R}$ interval cleaning & $3 d$ & $\begin{array}{l}\text { Artefact cleaning methods and percentage of } \\
\text { corrected beats }\end{array}$ \\
\hline HRV calculation & 4 & \\
\hline Method of analysis used & $4 a$ & $\begin{array}{l}\text { Metrics used and software for calculation, } \\
\text { log transform }\end{array}$ \\
\hline Frequency bands used & $4 b$ & $\begin{array}{l}\text { Specification of frequency bands and their } \\
\text { interpretation }\end{array}$ \\
\hline
\end{tabular}

HRV, heart rate variability.

HF power, and no differences were found between the two disorder groups. These results were also found to be independent of age, body mass index and medication. Moon et al, ${ }^{23}$ in 2013, measured HRV features in 41 patients with BD, 35 patients with schizophrenia, 34 patients with post-traumatic stress disorder (PTSD), 34 patients with major depressive disorder and 27 healthy controls, with the aim to discriminate between various mental health disorders using HRV. They found that it was not possible to discriminate between the disorders; however, it was possible to discriminate between the grouped mental health disorders and healthy controls through a reduction in a number of HRV features within the disorders, in particular the HF power component.

Faurholt-Jepsen et a ${ }^{24}$ compared HRV measures between 16 BD participants during different affect states. A significant increase in HRV was found during mania compared with both depression and euthymia, with no differences found between euthymia and depression. In addition to these differences, a negative correlation was found between severity of depressive symptoms and HRV. This suggests more severe depression is associated with reductions to HRV, with the opposite direction of correlation found for mania and HRV.

Most of the previous studies on HRV in mental health disorders require a short ECG recording under controlled conditions. The personalised monitoring systems for care in mental health (PSYCHE) project investigated the health of bipolar patients using a wearable monitoring system to record physiological signals, such as HRV, respiration and activity, and a smartphone monitoring systems to determine participant mood and send data to clinicians. ${ }^{1325-28}$ The project aimed to gain insight into the physiological and mood characteristics of patients with $\mathrm{BD}$ over longer periods in a naturalistic setting as opposed to the controlled environments of previous studies. An initial study included eight patients with BD, with over 400 hours of HRV data obtained through the wearable monitoring systems. The data were collected when a patient had been admitted to hospital and was recorded overnight, although no information is given about the length of time used for HRV analysis. Standard time and frequency domain measures were calculated: mRR, SDNN, RMSSD, pNN50, LF, HF and LF/HF ratio from the data, along with sample entropy. The HRV data were then used to classify subjects into one of four mood states (depression, mixed state, hypomania and euthymia) using support vector machines. The initial mood of each patient was assessed by a clinician, with changes in mood monitored by self-reported questionnaires; the mood states were then used to determine the accuracy of the classifiers, which use HRV to predict mood, with an accuracy of around $90 \% .{ }^{28}$ No data were collected on healthy controls (or another clinical group), making it difficult to establish the specificity of the findings to BD.

Other studies have investigated the effect therapy and stimulation has on HRV in people with BD. Howells et a ${ }^{29}$ studied $12 \mathrm{BD}$ and 9 healthy controls through frequency domain measures of HRV before and after cognitive behavioural therapy. Initially BD participants had elevated HF peaks. After the therapy, there were no differences between BD participants and the controls, suggesting the therapy improves emotional processing of the BD participants. Tanaka et al ${ }^{30}$ investigated how stimulation to the wrist affects frequency domain measures of HRV in 25 $\mathrm{BD}$ and 22 controls. No differences were found, although hormone levels were different in the groups, suggesting biological background did not influence these changes.

In general, HRV studies including BD participants find that there is a reduction in HRV compared with controls. However, with very few studies meeting all items on the GRAPH checklist (table 3) and with greatly varying methodologies across the studies, it is difficult to summarise the results in any greater detail.

\section{HRV in BPD}

Few studies have explored HRV in BPD. A study carried out by Austin et al investigated respiratory sinus arrhythmia (RSA) in nine patients with $\mathrm{BPD}$ and 11 healthy controls. Variations in R-R intervals due to respiration is known as RSA and is a measure of synchronicity of HRV and respiration rate and considered a marker of parasympathetic nervous system activity. The study showed significant difference in parasympathetic activity between patients with BPD and healthy controls through differences in RSA. ${ }^{31}$ Ebner-Priemer et al recorded 24 hours ECG signals on 50 patients with BPD and 50 healthy controls. ${ }^{32}$ HRV was calculated for the period at night at which the average HR was lowest; the results from the study tested the hypothesis that HRV is lower in BPD. However, the HF components of the HRV were found to be higher in patients with BPD, which is surprising as HF activity is related to parasympathetic activity. Furthermore, a study investigating parasympathetic and sympathetic activity through the use of RSA in 12 patients with BPD and 28 healthy controls had ECG recorded for three $5 \mathrm{~min}$ stages lat rest or stressed) found BPD was associated with lower values of RSA suggesting increased levels of sympathetic activity and decreased levels of parasympathetic activity. ${ }^{33}$ Meyer et al recorded 5 min ECG signals on 27 participants with BPD, 23 in remission from BPD, 18 suffering from PTSD and 23 healthy controls. ${ }^{34}$ Significant differences were only found between PTSD participants and controls; however, BPD participants had reduced variability across linear time and frequency domain measures compared with controls.

The lack of studies in people with BPD makes it difficult to draw conclusions about how HRV is altered in the disorder,especially as only two of the four studies included here use any of the standard HRV measures. Although these studies follow the GRAPH checklist relatively well (table 4), Ebner-Priemer and Meyer use ECG recordings over greatly varying timescales with differing results, with Meyer finding no differences between 

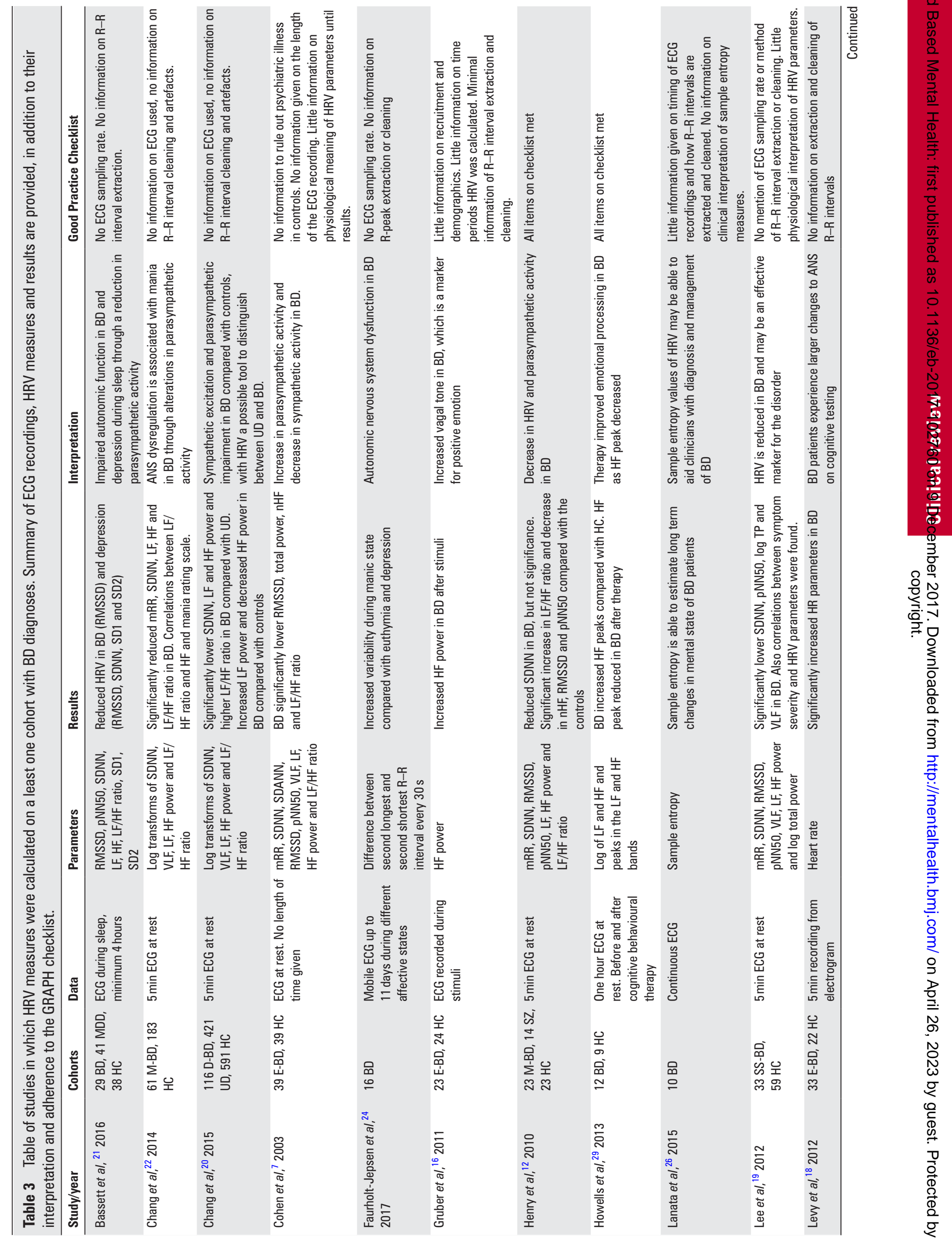


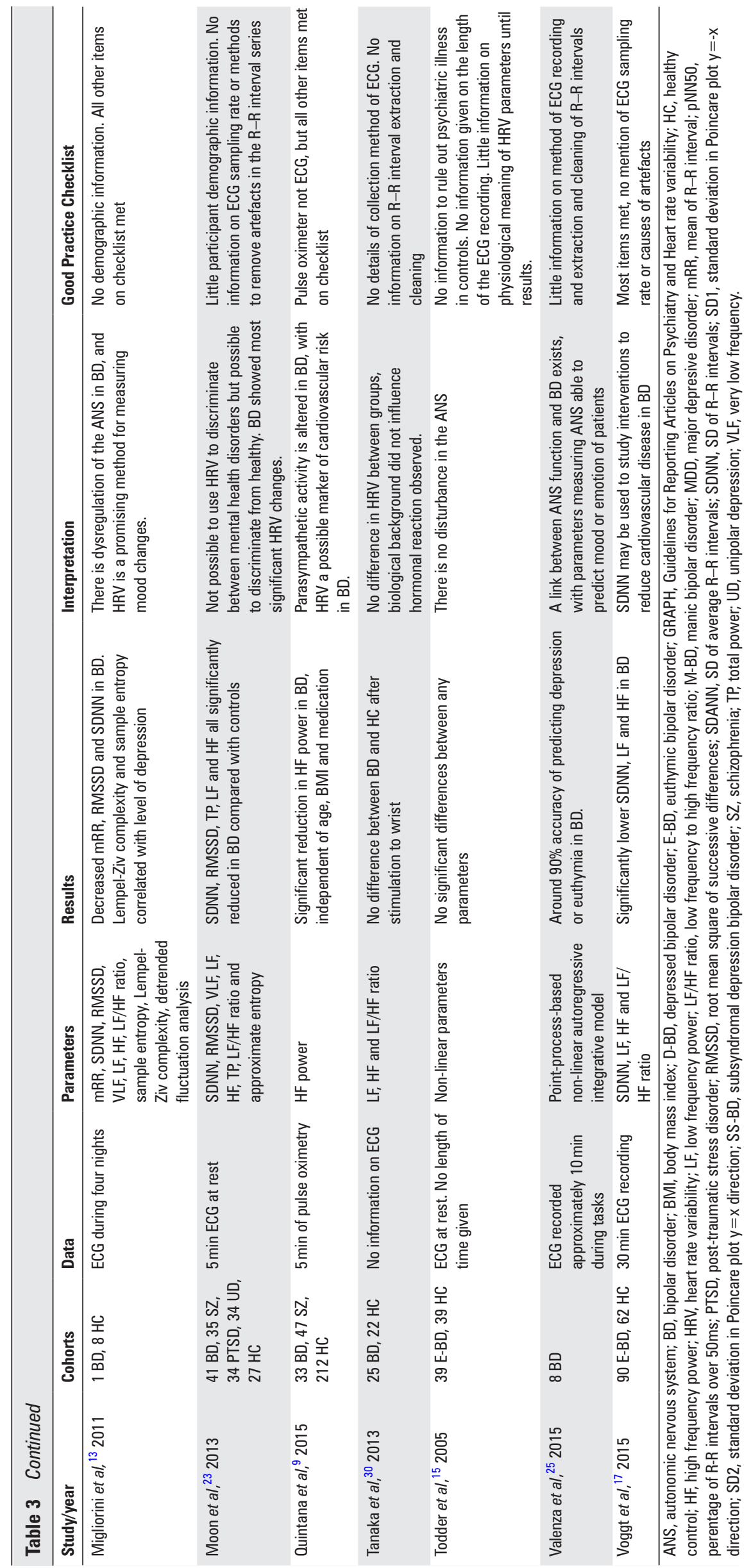

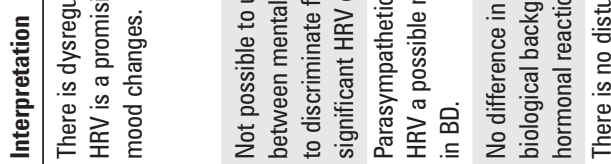


Table 4 Table of studies in which HRV measures were calculated on a least one cohort with BPD diagnoses. Summary of ECG recordings, HRV measures and results are provided, in addition to their interpretation and adherence to the GRAPH checklist.

\begin{tabular}{|c|c|c|c|c|c|c|}
\hline Study/year & Cohorts & Data & Parameters & Results & Interpretation & Good practice checklist \\
\hline $\begin{array}{l}\text { Austin et al, } \\
2007\end{array}$ & 9 BPD, 11 HC & $\begin{array}{l}\text { Four times } 10 \mathrm{~min} \\
\text { ECG watching } \\
\text { films }\end{array}$ & RSA & $\begin{array}{l}\text { Significantly reduced } \\
\text { RSA in BPD }\end{array}$ & $\begin{array}{l}\text { Lower RSA is linked to reduced } \\
\text { parasympathetic activity in BPD, } \\
\text { with changes in RSA much less after } \\
\text { emotional stimuli }\end{array}$ & $\begin{array}{l}\text { Little demographic information. HRV } \\
\text { not calculated }\end{array}$ \\
\hline $\begin{array}{l}\text { Ebner- } \\
\text { Priemer et al, } \\
2007\end{array}$ & $50 \mathrm{BPD}, 50 \mathrm{HC}$ & $\begin{array}{l}24 \text { hours } \\
\text { ambulatory ECG }\end{array}$ & $\begin{array}{l}\text { mRR, HF power, } \\
\text { HF power at } \\
\text { night }\end{array}$ & $\begin{array}{l}\text { Increased } m R R \text { and } \\
\text { HF power in BPD }\end{array}$ & $\begin{array}{l}\text { Increased HF power indicates } \\
\text { increased parasympathetic activity, } \\
\text { opposite to the expected findings }\end{array}$ & $\begin{array}{l}\text { Little demographic information. No } \\
\text { information on } \mathrm{R}-\mathrm{R} \text { interval extraction, } \\
\text { cleaning and dealing with artefacts }\end{array}$ \\
\hline $\begin{array}{l}\text { Meyer et al, } \\
2016\end{array}$ & $\begin{array}{l}27 \mathrm{BPD}, \\
23 \mathrm{BPD} \text { in } \\
\text { remission, } 18 \\
\text { PTSD, } 23 \mathrm{HC}\end{array}$ & $\begin{array}{l}5 \text { min at rest. } \\
\text { After emotional } \\
\text { face classification }\end{array}$ & $\begin{array}{l}\text { RMSSD, SDNN, } \\
\text { NN50, total } \\
\text { power, LF, HF } \\
\text { and LF/HF ratio }\end{array}$ & $\begin{array}{l}\text { HRV lower in all } \\
\text { groups compared with } \\
\text { HC. Only significant } \\
\text { for PTSD }\end{array}$ & $\begin{array}{l}\text { No difference in HRV between BPD and } \\
\text { HC. This may differ at varying stress } \\
\text { levels }\end{array}$ & $\begin{array}{l}\text { No ECG sampling frequency. R-R } \\
\text { interval extraction and cleaning using } \\
\text { Kubios, with no further detail }\end{array}$ \\
\hline $\begin{array}{l}\text { Weinberg et al, } \\
2009\end{array}$ & $12 \mathrm{BPD}, 28 \mathrm{HC}$ & $\begin{array}{l}\text { Three times } 5 \mathrm{~min} \\
\text { ECG }\end{array}$ & RSA & $\begin{array}{l}\text { Decreased RSA } \\
\text { values in } \mathrm{BPD}\end{array}$ & $\begin{array}{l}\text { Increased levels of sympathetic } \\
\text { activity and decreased levels of } \\
\text { parasympathetic activity indicated by } \\
\text { reduced RSA }\end{array}$ & $\begin{array}{l}\text { No information on disease } \\
\text { characteristics and little demographic } \\
\text { information. HRV not calculated. }\end{array}$ \\
\hline
\end{tabular}

$\mathrm{BPD}$, borderline personality disorder; HC, healthy control; HF, high frequency; HRV, heart rate variability; LF, low frequency; LF/HF ratio, low frequency to high frequency ratio; NN50, number of R-R intervals over 50ms; PTSD, post-traumatic stress disorder; RMSSD, root mean square of successive differences; RSA, respiratory sinus arrhythmia; SDNN, SD of $R-R$ intervals.

groups and Ebner-Premier finding increased parasympathetic activity in BPD, opposite to the expected result. More studies involving BPD participants are required which closely follow the GRAPH checklist before we can speculate how HRV is altered in BPD.

There have been no published studies where BPD and BD have been directly compared, making any conclusions tentative at best. The heterogeneity of methodologies employed in these studies adds a further level of complexity to any comparisons. At present there is insufficient evidence to allow comparison of HRV in BPD and BD to be made.

\section{CONCLUSION}

HRV is an important physiological marker in psychiatric illness and may provide important information about underlying phenotypes as well as cast light on the increased cardiovascular risk associated with psychiatric disorder. At present there is little consensus with respect to methodology that makes comparison difficult. The majority of previous studies on HRV in BD and BPD fail to meet every item on the GRAPH checklist. Interpretation of previous findings is difficult as there is often a lack of information available to accurately reproduce and compare results or build on previous work. If future studies were to closely follow this set of guidelines, it may accelerate HRV research in mental health and aid interpretation and reproducibility.

Given that diurnal rhythms disturbance is inherent in many psychiatric disorders and mobile ECG monitoring can now record signals for days at a time, diurnal patterns of HR and HRV measures may provide further insight into nervous system function. Future studies may investigate how sleep-wake cycles are linked to HRV and whether psychiatric disorders are associated with altered diurnal patterns of HRV.

Acknowledgements MdV is supported by EPSRC and Wellcome Trust Centre Grants 098461/Z/12/Z (Sleep, Circadian Rhythms and Neuroscience Institute). KS is supported by a Wellcome Trust Strategic Award 102616/Z (Collaborative Network for Bipolar Research to Improve Outcomes) and by the NIHR Oxford Health Biomedical Research Centre. OC acknowledges the support of an RCUK Digital Economy Programme grant number EP/G036861/1 (Oxford Centre for Doctoral Training in Healthcare Innovation)

Funding Wellcome Trust: 098461/Z/12/Z (Sleep, Circadian Rhythms and Neuroscience Institute); 102616/Z (Collaborative Network for Bipolar Research to Improve Outcomes).

Disclaimer The views expressed are those of the authors and not necessarily those of the NHS, the NIHR or the Department of Health.

Competing interests None declared.
Provenance and peer review Not commissioned; externally peer reviewed.

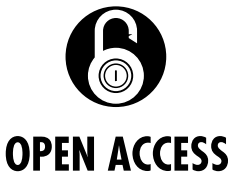

Open Access This is an Open Access article distributed in accordance with the terms of the Creative Commons Attribution (CC BY 4.0) license, which permits others to distribute, remix, adapt and build upon this work, for commercial use, provided the original work is properly cited. See: http://creativecommons.org/licenses/by/4.0/

doi:10.1136/eb-2017-102760

Received 15 June 2017; Revised 7 August 2017; Accepted 30 August 2017

\section{REFERENCES}

1. Alvares GA, Quintana DS, Hickie IB, et al. Autonomic nervous system dysfunction in psychiatric disorders and the impact of psychotropic medications: a systematic review and meta-analysis. J Psychiatry Neurosci 2016;41:89-104.

2. Quintana DS, Guastella AJ, Outhred T, et al. Heart rate variability is associated with emotion recognition: direct evidence for a relationship between the autonomic nervous system and social cognition. Int J Psychophysiol 2012;86:168-72.

3. Hansen $\mathbf{A L}$, Johnsen BH, Sollers JJ, et al. Heart rate variability and its relation to prefrontal cognitive function: the effects of training and detraining. Eur J Appl Physiol 2004;93:263-72.

4. Reinecke A, Filippini N, Berna C, et al. Effective emotion regulation strategies improve fMRI and ECG markers of psychopathology in panic disorder: implications for psychological treatment action. Trans/ Psychiatry 2015;5:e673-10.

5. Malpas SC. Neural influences on cardiovascular variability: possibilities and pitfalls. Am J Physiol Heart Circ Physiol 2002;282:H6-20.

6. Sztajzel J. Heart rate variability: a noninvasive electrocardiographic method to measure the autonomic nervous system. Swiss Med Wkly 2004;134:514-22.

7. Cohen H, Kaplan Z, Kotler M, et al. Impaired heart rate variability in euthymic bipolar patients. Bipolar Disord 2003;5:138-43.

8. Nardelli M, Valenza G, Cristea IA, et al. Characterizing psychological dimensions in non-pathological subjects through autonomic nervous system dynamics. Front Comput Neurosci 2015;9:37.

9. Quintana DS, Westlye LT, Kaufmann T, et al. Reduced heart rate variability in schizophrenia and bipolar disorder compared to healthy controls. Acta Psychiatr Scand 2016;133:44-52.

10. Francesco B, Maria Grazia B, Emanuele G, et al. Linear and nonlinear heart rate variability indexes in clinical practice. Comput Math Methods Med 2012;2012:1-5.

11. Reyes del Paso GA, Langewitz W, Mulder LJ, et al. The utility of low frequency heart rate variability as an index of sympathetic cardiac tone: a review with emphasis on a reanalysis of previous studies. Psychophysiology 2013;50:477-87.

12. Henry BL, Minassian A, Paulus MP, et al. Heart rate variability in bipolar mania and schizophrenia. J Psychiatr Res 2010;44:168-76.

13. Migliorini M, Mendez MO, Bianchi AM. Study of Heart Rate Variability in Bipolar Disorder: Linear and Non-Linear Parameters during Sleep. Front Neuroeng 2011;4:22. 
14. Quintana DS, Alvares GA, Heathers JA. Guidelines for Reporting Articles on Psychiatry and Heart rate variability (GRAPH): recommendations to advance research communication. Trans/ Psychiatry 2016;6:e803-10

15. Todder D, Bersudsky $Y$, Cohen $\mathrm{H}$. Nonlinear analysis of RR interval in euthymic bipolar disorder. Auton Neurosci 2005;117:127-31.

16. Gruber J, Dutra S, Eidelman P, et al. Emotional and physiological responses to normative and idiographic positive stimuli in bipolar disorder. J Affect Disord 2011;133:437-42.

17. Voggt A, Berger $M$, Obermeier $M$, et al. Heart rate variability and Omega-3 Index in euthymic patients with bipolar disorders. Eur Psychiatry 2015;30:228-32

18. Levy B. Autonomic nervous system arousal and cognitive functioning in bipolar disorder. Bipolar Disord 2013;15:70-9.

19. Lee JS, Kim B, Hong Y, et al. Heart rate variability in the subsyndromal depressive phase of bipolar disorder. Psychiatry Clin Neurosci 2012;66:361-6.

20. Chang HA, Chang CC, Kuo TB, et al. Distinguishing bipolar II depression from unipolar major depressive disorder: Differences in heart rate variability. World J Biol Psychiatry 2015; 16:351-60.

21. Bassett D, Bear N, Nutt D, et al. Reduced heart rate variability in remitted bipolar disorder and recurrent depression. Aust N Z J Psychiatry 2016;50:793-804.

22. Chang HA, Chang CC, Tzeng NS, et al. Heart rate variability in unmedicated patients with bipolar disorder in the manic phase. Psychiatry Clin Neurosci 2014;68:674-82

23. Moon E, Lee SH, Kim DH, et al. Comparative Study of Heart Rate Variability in Patients with Schizophrenia, Bipolar Disorder, Post-traumatic Stress Disorder, or Major Depressive Disorder. Clin Psychopharmacol Neurosci 2013;11:137-43.

24. Faurholt-Jepsen M, Brage S, Kessing LV, et al. State-related differences in heart rate variability in bipolar disorder. J Psychiatr Res 2017;84:169-73.
25. Valenza G, Citi L, Gentili C, et al. Characterization of depressive States in bipolar patients using wearable textile technology and instantaneous heart rate variability assessment. IEEE J Biomed Health Inform 2015;19:263-74.

26. Lanata A, Valenza G, Nardelli M, et al. Complexity index from a personalized wearable monitoring system for assessing remission in mental health. IEEE J Biomed Health Inform 2015;19:132-9.

27. Valenza G, Citi L, Lanatá $A$, et al. Revealing real-time emotional responses: a personalized assessment based on heartbeat dynamics. Sci Rep 2014:4:4998.

28. Valenza G, Nardelli M, Lanatà A, et al. Wearable monitoring for mood recognition in bipolar disorder based on history-dependent long-term heart rate variability analysis. IEEE J Biomed Health Inform 2014;18:1625-35

29. Howells FM, Laurie Rauch HG, Ives-Deliperi VL, et al. Mindfulness based cognitive therapy may improve emotional processing in bipolar disorder: pilot ERP and HRV study. Metab Brain Dis 2014;29:367-75.

30. Tanaka Y, Maruyama Y, Ishitobi Y, et al. Salivary alpha-amylase and cortisol responsiveness following electrically stimulated physical stress in bipolar disorder patients. Neuropsychiatr Dis Treat 2013;9:1899-904.

31. Austin MA, Riniolo TC, Porges SW. Borderline personality disorder and emotion regulation: insights from the Polyvagal Theory. Brain Cogn 2007:65:69-76.

32. Ebner-Priemer UW, Welch SS, Grossman P, et al. Psychophysiological ambulatory assessment of affective dysregulation in borderline personality disorder. Psychiatry Res 2007; 150:265-75

33. Weinberg A, Klonsky ED, Hajcak G. Autonomic impairment in borderline personality disorder: a laboratory investigation. Brain Cogn 2009;71:279-86.

34. Meyer PW, Müller LE, Zastrow A, et al. Heart rate variability in patients with posttraumatic stress disorder or borderline personality disorder: relationship to early life maltreatment. J Neural Transm 2016;123:1107-18. 